\title{
TOWARDS PREDICTION AND CONTROL OF LARGE SCALE TURBULENT STRUCTURE SUPERSONIC JET NOISE
}

\author{
Robert H. Schlinker* \\ United Technologies Research \\ Center \\ E. Hartford, CT, USA
}

Tim Colonius

California Institute of Technology

Pasadena, CA, USA

\author{
Ramons A. Reba \\ United Technologies Research \\ Center \\ E. Hartford, CT, USA
}

\author{
Kristjan Gudmundsson \\ California Institute of Technology \\ Pasadena, CA, USA
}

\author{
John C. Simonich \\ United Technologies Research \\ Center \\ E. Hartford, CT, USA
}

\author{
Foluso Ladeinde \\ TTC Technologies \\ Centereach, NY, USA
}

\begin{abstract}
In this paper, we report on progress towards developing physics-based models of sound generation by large-scale turbulent structures in supersonic jet shear layers generally accepted to be the source of aft-angle noise. Aside from obtaining better engineering prediction schemes, the development and optimization of long term jet noise reduction strategies based on controlling instability wave generated largescale turbulence structures in the shear layer can be more successful if based on predictive flow-noise models, rather than on build and test approaches alone. Such models, if successful, may also provide a path by which laboratory scale demonstrations can be more reliably translated to engine scale. Results show that the noise radiated by large-scale structures in turbulent jet shear layers may be modeled using a RANS based PSE method and projected to the far-field using a Kirchhoff surface approach. A key enabler in this procedure is the development of near-field microphone arrays capable of providing the pressure statistics needed to validate the instability wave models. Our framework provides, for the first time, a deterministic model that will allow understanding and predicting noise radiated by large-scale turbulence.
\end{abstract}

\subsection{INTRODUCTION}

\subsection{Motivation}

In contrast to military engines, jet exhaust noise for subsonic commercial aircraft has been dramatically reduced in recent decades as a result of increased bypass ratio (BPR). However, for military applications, the requirement of high specific thrust generally precludes such a cycle-based strategy. Specifically, commercial aircraft typically have $\mathrm{BPR}>2$ to dramatically reduce jet exhaust velocity and, hence, noise. These conditions cannot be matched by military engines with BPR $<$ 1.2 to minimize the impact on thrust. As an example, to achieve a $10 \mathrm{dBA}$ benefit would require approximately a $25 \%$ decrease in specific thrust based on a simple $8^{\text {th }}$ power velocity scaling law for jet noise generation. Thus, highly effective subsonic jet noise reduction schemes do not transition well to the supersonic regime and tactical military aircraft performance requirements impose severe constraints on the available options for noise reduction. As a result, the high noise levels experienced by aircraft carrier launch/recovery crews as well as communities in close proximity to operational air bases have stimulated renewed research interest in component-level technologies for supersonic jet noise suppression.

Current component level retro-fitable technologies being investigated for low BPR supersonic jets are providing benefits of 2-4 $\mathrm{dBA}$ in the near term. Approaches range from mechanical chevrons [1-6], gaseous and liquid injection [7], fluidic chevrons $[4,5,8,9]$, and lobed inserts creating corrugations on the interior of the nozzle perimeter [3]. Noise reduction may occur due to break-up of shock cells in overexpanded flows and/or by reducing turbulent mixing noise by

\footnotetext{
* Project Leader and author of correspondence
} 
the same mechanisms for which these devices have proven successful in subsonic jets.

More dramatic reductions in supersonic jet noise will likely require a multi-faceted approach combining new technologies for mixing noise reduction and control of shock-associated noise through isentropic nozzle design and variable throat-toexit diameter to compensate for different operating conditions over the flight envelope. New concepts such as asymmetric or beveled nozzles have been investigated $[10,11]$ to create asymmetric flow fields that modify shock structures and shear layer mixing. Thermal acoustic fluid shielding $[12, \mathbf{1 3}, \mathbf{1 4}]$, involving addition of a second heated stream on the nozzle perimeter to trap high frequency noise generation inside the jet plume, has also been investigated. Recent studies using this concept routed engine bypass air to a semi-annular fluid shield on the underside of the engine [5]. Also showing promise are active control strategies [16-19] relying on the forced response of the near-nozzle shear layer. The impact on engine and aircraft performance, weight and survivability remains to be considered for all of these approaches.

In this paper, we report on progress towards developing physics-based models of sound generation by large-scale turbulent structures in supersonic jets. Aside from obtaining better engineering prediction schemes, we argue that development and optimization of the aforementioned control strategies can be more successful if based on predictive noise models, rather than on build and test approaches alone. Such models, if successful, may also provide a path by which laboratory scale demonstrations can be more reliably translated to engine scale.

While jet noise prediction based on Large Eddy Simulation (LES) has made significant strides in recent years (see [20] for a review), the associated computational expense is significant, and precludes routine use in engineering applications. Aside from LES, virtually all noise prediction methods rely on the acoustic analogy with statistical models for the equivalent sources. Such models had, until recently, failed to adequately predict the acoustic field at aft angles where large-scale structure noise dominates. Recently, increasingly complex forms of the acoustic analogy [21] and improved noise source models have led to improved predictions for aft angles at a few operating conditions [22-24]. However, the turbulent correlations for these models remain elusive to direct measurement in high-speed jets, and, in any event the universality of the correlations across jet operating conditions and complex nozzle designs has not been proven. Also, the acoustic analogy equivalent sources have not provided insights into the mechanisms by which noise from large scale structures may be reduced. Thus, the current research program aims to develop computationally inexpensive noise prediction tools based on reduced-order descriptions of the acoustic sources.

\subsection{Supersonic jet noise mechanisms}

To set the context for the modeling effort discussed in this paper, we briefly summarize the relevant noise mechanisms in military jet exhausts. Key mechanisms are shock-associated noise, large-scale and fine-scale mixing noise, and augmentor noise. For a more exhaustive review of the first three mechanisms, see Tam [25]. Although many more publications by other authors area available, the discussion here is intended only to set the context.

Shock-associated noise is generated when the nozzle is nonideally expanded, creating shock cells in the exhaust plume (Figure 1a). Interaction of the shear layer turbulence with the shock cells generates broadband noise. The resulting shock noise radiates in the forward direction, and is a significant contributor at polar angles near $90^{\circ}$ to the engine inlet axis (inlet at $0^{\circ}$ ), but it is of less significant at aft angles $\left(>120^{\circ}\right)$ where jet-mixing noise peaks. A special situation occurs if significant acoustic energy is radiated directly upstream $\left(0^{\circ}\right)$. This energy can create a feedback loop between the nozzle/airframe components exciting the existing shear layer instability wave, resulting in a discrete tone known as jet screech.

The second and third jet noise sources are linked to the high levels of turbulence associated with the jet flow. One of these sources is related to large-scale turbulent structures that advect and evolve in the near nozzle shear layer. The genesis of these structures, even for initially turbulent shear layers [26], is related to basic instabilities of the shear-layer velocity profile, and, we refer to them throughout this paper as wave packets (Figure 1b). When these structures have supersonic convection velocities, they are capable of directly radiating to the far-field by analogy with supersonic flow over a wavy wall [25]. Since their advection velocity is some fraction of the jet Mach number, they do not radiate directly until the jet Mach number is well into the supersonic range, but, even at subsonic speeds, the wave packets radiate sound because their envelope grows, saturates, and decays. At the end of the potential core, the non-linear breakdown and interaction of the wave packets generates further mixing noise.

The sound radiated by these large-scale structures controls the aft directivity peak and represents the most energetic component of the turbulent mixing noise. For comparison, shock-associated noise can be significant in non-ideally expanded jets, but, peaks in the forward arc ( $<90 \mathrm{deg})$; thus, any reductions in shock noise will minimally impact the aft noise levels controlled by the organized turbulence structure.

The third jet exhaust noise source is generated by the small scale, or fine scale, turbulence (Figure 1c). The noise directivity is controlled by refraction in the shear layer, caused by velocity and temperature gradients. This mechanism peaks 


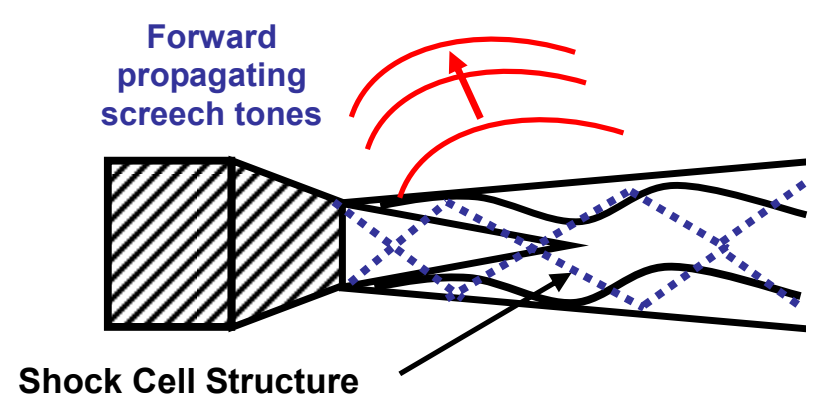

a) Shock-Shear Layer Interaction Noise

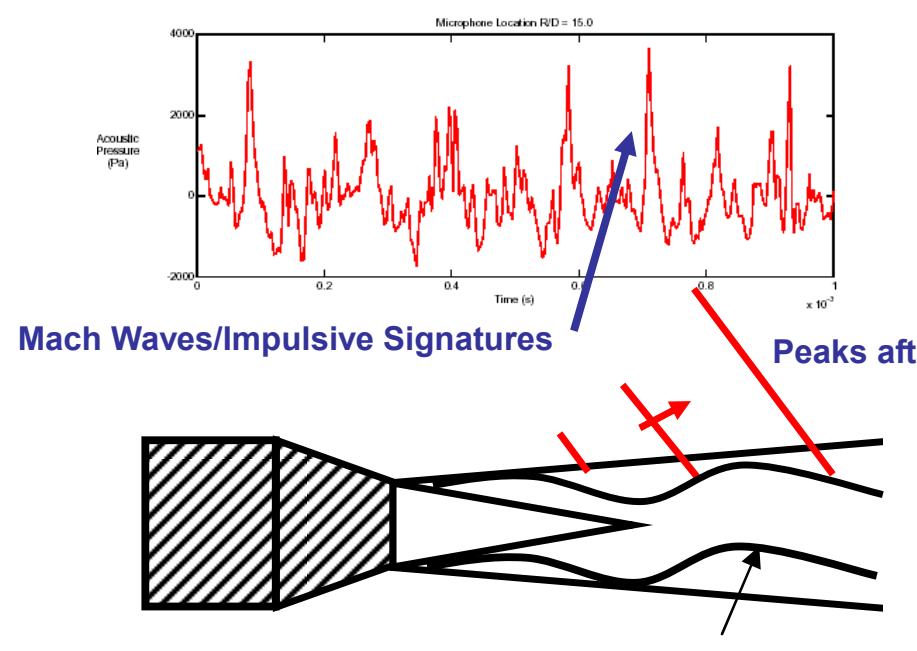

Instability wave/wave packet

\section{Other Mechanisms}

- Combustion noise is weak

- Jet in cross flow intensifies noise

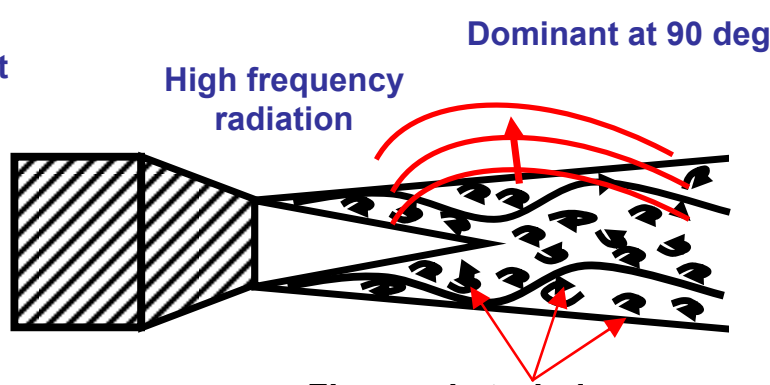

Fine-scale turbulence

\section{b) Organized Turbulence Structure Noise}

c) Fine Scale Turbulence Noise

Figure 1. Supersonic jet noise mechanisms

at $90^{\circ}$ from the inlet axis, and is weaker than the large-scale structure noise at the aft peak directivity angles of $\sim 135-140^{\circ}$ for military engines. The noise directivity is controlled by the acoustic wave's refraction in the shear layer, caused by velocity and temperature gradients. Refraction bends the acoustic waves off the jet center line resulting in a narrow region around the jet center line that has much lower noise levels. This area has become known as the cone of silence. In supersonic jets this area is not as prominent because of additional Mach wave radiation that still radiates quite strongly to extreme aft angles.

In reality, the turbulent motions occur over a continuous range of scales, and result in broadband noise. However, experimental evidence suggests that the small and large-scales result in two essentially distinct spectra, as illustrated in
Figure 2, based on the analysis of Tam et al. [27] and Tam [28] using a large collection of model scale subsonic and supersonic jet noise data. For the organized structure noise (Figure 2a), an empirical spectrum fit function, $F$, with amplitude and a spectral peak coefficient is capable of representing the measured aft radiation at various jet Mach numbers $\left(\mathrm{M}_{\mathrm{j}}\right)$, temperature ratios $\left(\mathrm{T}_{\mathrm{r}}\right)$, and polar directivity angles (alp). The raw data is represented by the "squiggly" trace while the fit function is given by the smooth line. The test conditions are: a) $\mathrm{M}_{\mathrm{j}}=2.0, \mathrm{~T}_{\mathrm{r}}=4.89$, alp=160 deg; b) $\mathrm{M}_{\mathrm{j}}=2.0, \mathrm{~T}_{\mathrm{r}}=1.11$, alp=160 deg; c) $M_{j}=1.96, T_{r}=1.78$, alp=138.6 deg; d) $M_{j}=$ $1.49, \mathrm{~T}_{\mathrm{r}}=1.11$, alp=138.6 deg. 


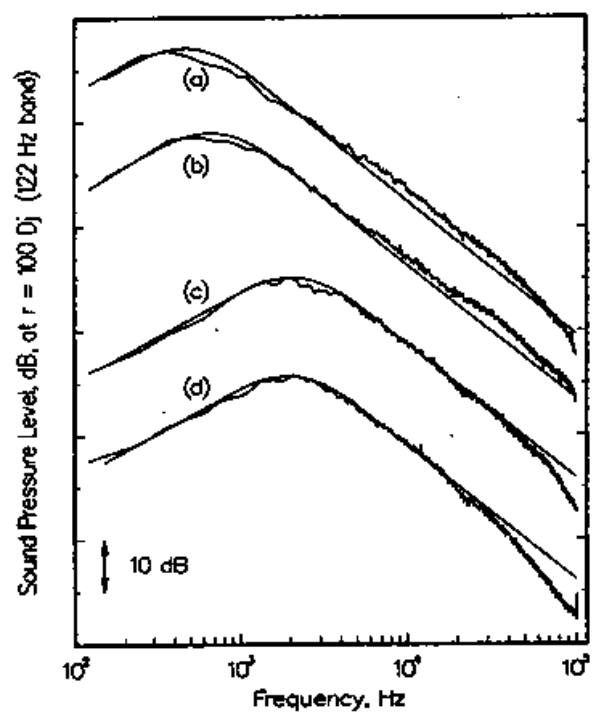

a) Aft angles $>90 \mathrm{deg}$.

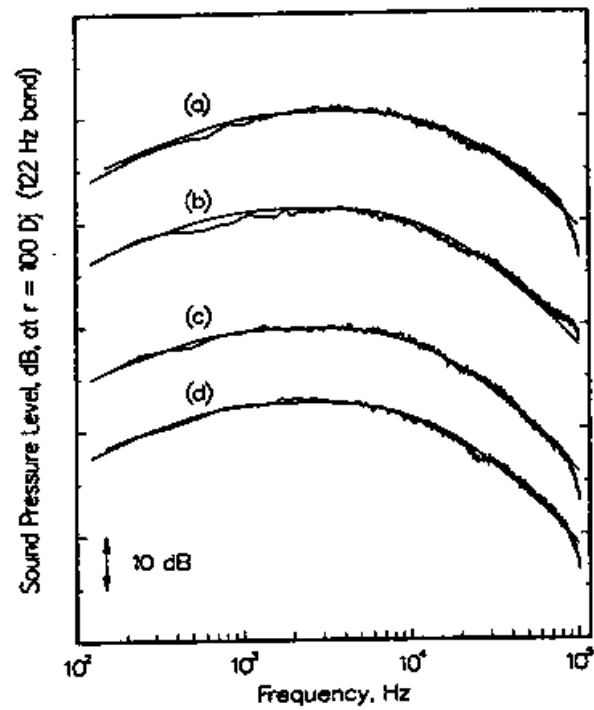

b) Upstream angles $<90 \mathrm{deg}$.

Figure 2. Spectra at aft and forward angles showing universality of spectrum shape for organized turbulence structure noise and fine scale noise

Similarly, the fine scale turbulence (Fig. 2b) is well represented by the Tam derived $\mathrm{G}$ function fit for another data set: a) $\mathrm{M}_{\mathrm{j}}=$ $1.49, \mathrm{~T}_{\mathrm{r}}=2.35$, alp=92.9 deg; b) $\mathrm{M}_{\mathrm{j}}=2.0, \mathrm{~T}_{\mathrm{r}}=4.89$, alp=83.8 deg; c) $\mathrm{M}_{\mathrm{j}}=1.96, \mathrm{~T}_{\mathrm{r}}=0.99$, alp=83.3 deg; d) $\mathrm{M}_{\mathrm{j}}=1.96, \mathrm{~T}_{\mathrm{r}}=$ 0.98 , alp=120.2 deg. Overall, these functional forms can be used to estimate jet noise levels at different operating conditions and polar directivity angles.

The final mechanism we consider is the internal broadband combustion noise generated by fuel addition in the exhaust nozzle. Here we are focusing on the non-screech condition. The resulting broadband noise has been postulated by some researchers to be a potentially important contributor to peak directivity in aft quadrant, since noise levels typically increase with fuel addition. However, phased array measurements on a full scale engine [15] show that the source distributions peak at 5-10 diameters downstream of the nozzle exit for all frequencies whether in the full power or fuel addition mode. Thus, the observed noise increase in the fuel addition mode is due to fluid mechanics noise mechanisms rather than internal combustion noise or convection of entropy hot spots through turbine and nozzle components. Hence, engine internal "excess" noise is not viewed as a dominant mechanism.

\subsection{Long Term Program Goal and Roadmap}

In the long term, our research effort aims to develop component-level technologies based on controlling instability wave generated large-scale turbulence structures in the jet shear layer, generally accepted to be the source of aft-angle noise. Noise control strategies are envisioned based on modifying the instability wave spatio-temporal structure by introducing unsteady forcing in the initial shear layer region. Since this region is highly receptive to perturbations, the actuator authority and power requirements will be significantly lower compared to requirements for steady forcing reported in prior studies. While this concept is not new, its development and impact on noise reduction has been hindered by the lack of physics-based forcing and control strategies. Therefore, a key objective of the current program is to develop fundamental knowledge and innovative modeling to identify such strategies.

The current large-scale noise methodology development has multiple elements. First, we develop models to predict the nearfield pressures associated with large-scale turbulent structures (wave packets). These models are based on representations as instability waves evolving in the turbulent mean flow field, and are discussed in detail in Section 3.1 and 3.2. In order to develop an integrated noise model, we show how these models can be based on RANS descriptions of the mean flow field. In Section 3.3, we report on techniques to project the near-field pressures, via solution of the wave equation, to the far-field, thus developing a quantitative cause-and-effect relationship 
between perturbations to the shear layer and far-field sound. Finally, in Section 3.4, we discuss plans for future studies that combine these models with measured and implied near-nozzle disturbance spectra, and actuator inputs, to serve as a model for active noise reduction.

As part of the first and second elements, a unique experimental diagnostic technique was developed to detect the unsteady pressure signatures of organized turbulent structures at the interface between the jet flow and the acoustic radiation field. This region, located in the jet hydrodynamic near field, is viewed as the sound "source" containing the traveling wave pressure signature responsible for noise radiating to the far field in the aft direction. The source field can be measured with microphones just outside the non-linear turbulent flow region providing spectral features and length scales of the large-scale turbulence noise sources. As will be described later, these directly measured features become the basis of a convected wave packet model that has successfully linked the near field source with the acoustic far field. The experimental technique is discussed in Section 2.

\subsection{EXPERIMENTAL APPROACH}

Numerous experimental approaches for inferring sound sources have been proposed over the years. These include measurements of far-field sound and in-flow density or velocity fluctuations aiming at establishing direct cause-and-effect relationship between turbulence characteristics and noise. Other approaches have focused on near-field pressure. A summary of the early approaches is given in [29]. While these studies represent significant advances toward revealing noise mechanisms in high-speed jets, they were unable to (quantitatively) project sound levels in the far-field using measured near-field source information.

The experimental approach is based on the concept of measuring pressure statistics just outside the jet shear layer to capture signatures of large-scale structures/instability waves. The first experimental-diagnostic method linking near-field source measurements and far-field sound was presented by Reba et al. [30]. That study was limited to subsonic jets, and utilized near-field array data acquired by Suzuki and Colonius [31] in partnership with NASA Glenn Research Center. In the present paper, we describe a second generation diagnostic array (built on the methods in [31]), and show its success when applied to an $\mathrm{Mj}=1.5$ jet.

Microphones are configured to be sufficiently near the turbulent flow region to capture flow structure signatures, yet far enough that non-linear source terms can be neglected. Under these conditions, the linear wave equation describes both the flow structure signatures (the source) and the acoustic wave propagation. Therefore the linear wave equation can be used to "project" the source pressure to the acoustic field (to the extent that non-linear propagation effects are not important), and thereby relate dynamics of large-scale turbulence to noise generation. This observation is essential, since the diagnostic method aims to establish quantitative cause-and-effect relationships between dynamics of organized structures and farfield sound, and not merely to project sound from one location to another. The currently used projection method, described by Reba in [29], was enhanced since the initial approach reported in [30].

Experimental studies were conducted in the United Technologies Research Center (UTRC) Acoustic Research Tunnel (ART) developed in 1970 as the first forward flight anechoic simulation facility for jet noise, fan and propeller noise, and lifting surface/airframe noise studies. A recent description is given by Simonich et al. [32]. The facility provides up to a 50 " open jet forward flight simulation for jet noise using large single stream exhaust nozzles up to 6" diameter to achieve relevant Reynolds numbers, stagnation temperatures up to $1800 \mathrm{deg} \mathrm{R}$, and Mach numbers (M) up to 2.5 based on the 400 psi supply air. In the current study, the 3" diameter round supersonic nozzle was designed by method of characteristics to provide ideal expansion or shock free flow at the nozzle exit.

The exhaust nozzles project beyond the open jet as shown in Figure 3. Boundary layer suction exists on the model nozzle exterior to control boundary layer thickness for simulation of full-scale engine nacelle external flows. The free field microphones located outside the open jet flows $(\mathrm{M}=0.1$ to 0.36$)$ provide sound pressure level measurements over the key jet noise directivity angles ranging from $80 \mathrm{deg}$ to $155 \mathrm{deg}$ from the engine inlet centerline. Higher Mach numbers up to $\mathrm{M}=0.6$ are available with a 21 "x 31 " open jet test section.

The test section is surrounded by a sealed anechoic chamber 16 feet high, $18 \mathrm{ft}$ long (in the jet centerline direction) and $22 \mathrm{ft}$ wide. The chamber walls are lined with 18 inch deep fiberglass wedges which provide an anechoic acoustic environment above $175 \mathrm{~Hz}$. Downstream of the test section, the open jet air flow enters a diffuser through a circular collector with acoustic treatment on its flow impingement surface. The diffuser is designed to operate unstalled and hence is not a major source.

The jet is supplied with high pressure air from a compressor system capable of delivering $20 \mathrm{lb} / \mathrm{sec}$ of dry air continuously. The air is heated using a propane SUE burner. Flow from the combustor passes through a muffler which attenuates upstream combustion and valve noise. The airflow rate and combustor 

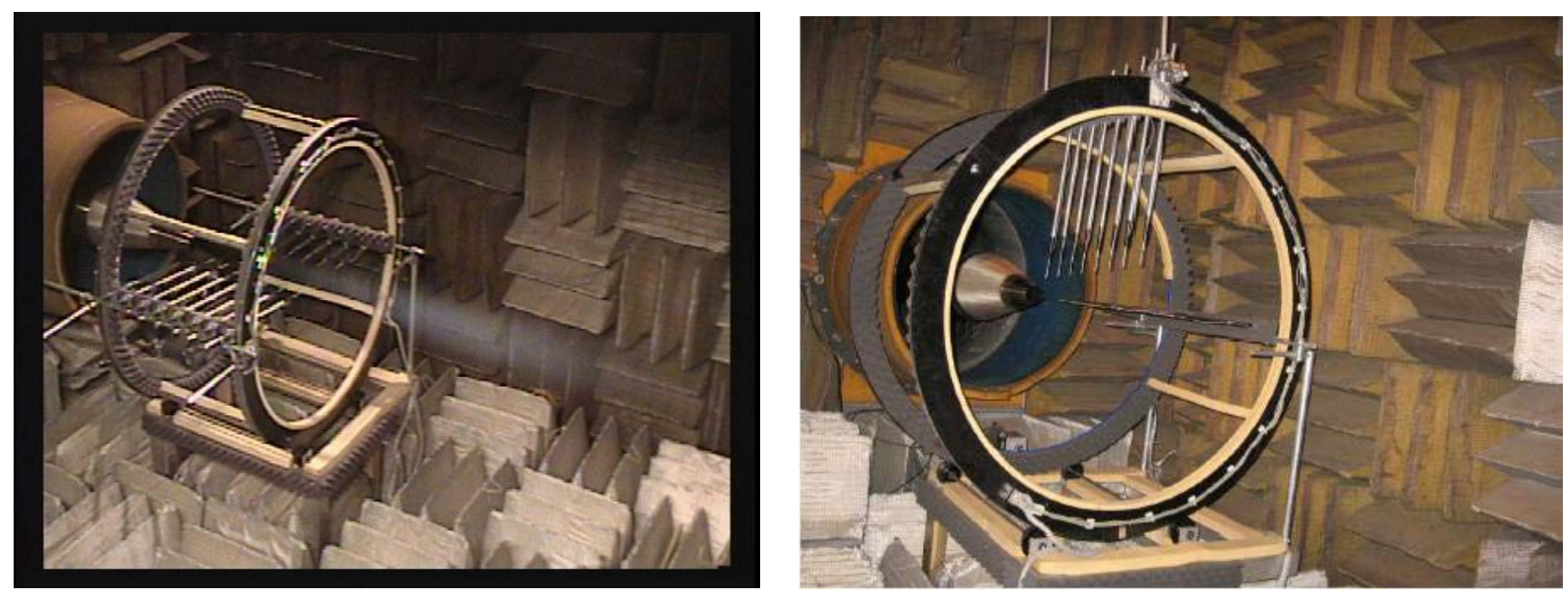

Figure 3. Rotating array installed in UTRC ART: a) supersonic jet noise study, b) view looking upstream into open jet with rotating array in vertical position

temperature are regulated by a programmed logic controller (PLC). Airflow is precisely controlled by using two valves in parallel. The larger valve is used to set the high flow rate and the small valve is used for precision control.

Near-field pressure measurements were acquired using a novel rotating array concept specifically designed to facilitate diagnostic studies of complex nozzle geometries of technological interest (e.g. chevron nozzles). The concept uses two linear microphone arrays, as visible in Figure 3. One linear array can be rotated automatically in the azimuthal direction. The second array (i.e. the reference array) is also movable, but requires manual adjustment. For a given position of the reference array, phase-locked data between the two arrays is acquired for each location of the movable azimuthal array (the number of locations is governed by the desired azimuthal modal resolution). With this approach, the modal content at any axial location is determined by Fourier transformation of the two-point azimuthal correlation.

A key design intent of this array is to facilitate application to complex nozzle geometries by minimizing the required microphone count, and maximizing adjustability in terms of axial location and streamwise extent. In particular, this approach minimizes the number of microphones needed to resolve higher azimuthal modes, which can become a source of aliasing error in complex non-circular nozzles, or in jets with shear-layer excitation at higher modes. Details of the rotating mechanical components are given in $[29,33]$.

For the results reported here, 8 microphones were used on each linear array, encompassing approximately the first 10 jet diameters. Microphones were spaced axially by $1.25 D_{j}$, with a spreading angle (cone half- angle) of \pm 7 degrees. The first microphone was located at $1.13 D_{j}$ from the nozzle exit, at a radial distance of $0.97 D_{j}$ from the jet centerline. The downstream-most microphone was located at $9.88 D_{j}$, at a radial distance of $2.05 D_{j}$. The axial extent of the array can be readily increased beyond the support rings by lengthening the microphone support rods. The 16 microphone signals were simultaneously sampled using a DataMax DTX-9R data acquisition system at a $100 \mathrm{kHz}$ sampling rate. The phase response of each microphone was calibrated to $30 \mathrm{KHz}$ using a Bruel \& Kjaer Type 9721 microphone calibrator.

Following these early experiments, the diagnostic array was increased to 22 microphones so that the downstream-most microphone is located at $12 D_{j}$. Sampling rate was increased to $500 \mathrm{KHz}$ to better capture Mach wave like impulsive signatures. Further increases in microphone number are planned in 2009.

\subsection{MODELING APPROACH AND VALIDATION}

\subsection{Wave Packet Models}

As discussed in Section 1.1, difficulties associated with modeling turbulent sound sources with the acoustic analogy approach have motivated an alternative approach to predicting noise from large-scale structures. The method relies on 

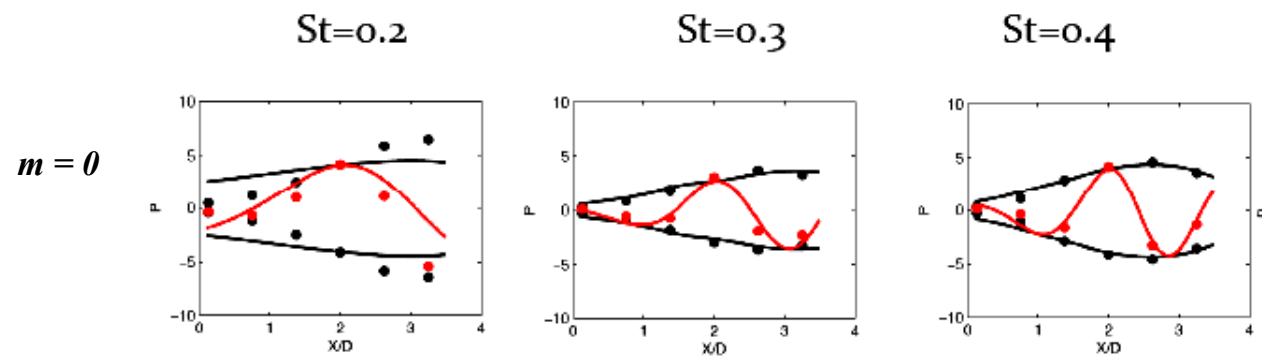

$\mathrm{St}=0.5$
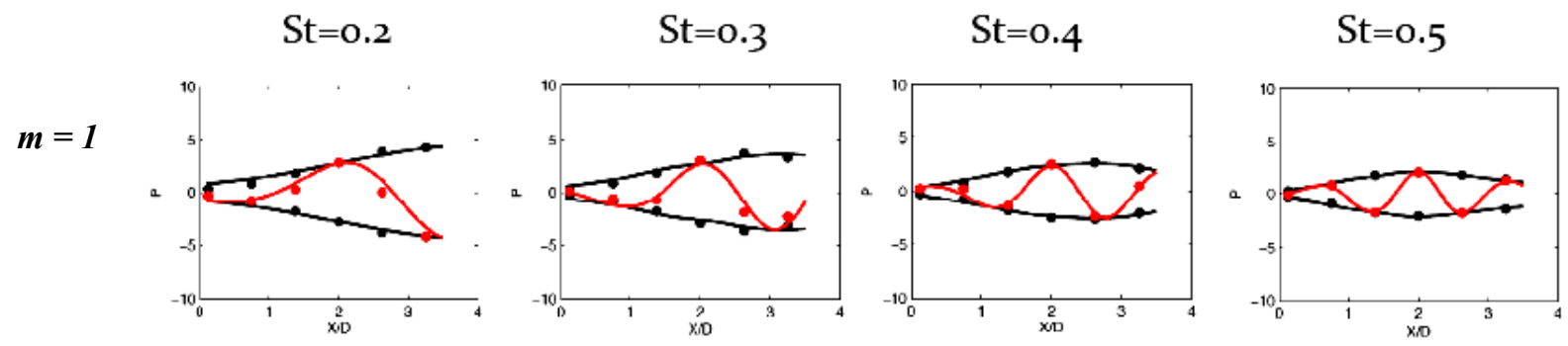

Figure 4. Comparison of pressure $(\mathrm{Pa})$ wave packet amplitudes from microphone (symbols) and linear instability wave theory (lines).

predicting, as a function of frequency, the convecting pressure wave packet that is associated with large-scale structures in the turbulent flow, and can be directly observed, via microphone measurements, in a region just outside the turbulent shear layers. This region is defined as that being sufficiently removed from the shear layers so that non-linear interactions are negligible, while simultaneously being sufficiently close so that, for subsonically convecting disturbances, the pressure field is evanescent, or exponentially decaying, with a convective rather than acoustic phase speed. Provided that pressure fluctuations from smaller-scale turbulence in the shear layer decay sufficiently fast, the existence of such a evanescent wave region is predicted from quasi-parallel stability theory of jet mean flow fields, and was initially confirmed in detail through the use of a caged 78 microphone array for a variety of jet operating points and for both round [31] and chevron nozzles [54]. At supersonic convection velocities there is direct radiation from these wave packets in the form of Mach waves $[34,35]$, but the overall structure of the wave packet is not qualitatively different from subsonic convection velocities.

It is important to note that we have so far avoided referring to the wave packets as instability waves. For many years, researchers have associated large-scale turbulent structures in free shear layers with instability waves of laminar and turbulent mean flow fields [36-44]. However, attempts to model features of jet mixing and noise radiation from these instability models have been largely unsuccessful, excepting forced shear flows. Another exception is at supersonic jet speeds, where features of Mach wave radiation have successfully been predicted using a
Matched Asymptotic Expansion between the quasi-parallel instability wave in the near-field and solutions of the wave equation in the far-field [34]. A key difference between the current approach and previous work is that we avoid attempting to equate measured flow quantities inside the turbulent flow with instability waves. Any such identification is problematic since flow quantities inside the region of intense turbulence are comprised of a variety of temporal and spatial scales.

On the other hand, outside the shear layer where smaller-scale pressure fluctuations are absent, it appears that the observed pressure wave-packet structure can, at least before the end of the potential core, be directly associated with linear instability waves (of the turbulent mean flow field). The evidence for this was provided by Suzuki and Colonius [31], where instability wave amplitudes were computed from experimentally determined mean flow fields (based on PIV measurements by Bridges et al. $[\mathbf{4 5}, \mathbf{4 6}]$ ) and compared directly to pressure signals from the 78 microphone array. A sample of the results from [31] is shown in Figure 4 for the first two azimuthal modes, $\mathrm{m}=0$ and $\mathrm{m}=1$. The data corresponds to a turbulent round jet ( $M=0.5$, cold) for axial stations up to $x / D=4$. The black lines indicate the amplitude of the wave packet; the red lines indicate the real part when the phase has been set to zero at the $4^{\text {th }}$ microphone position. The amplitude for the quasiparallel linear theory is selected to give the best agreement with the data, in a least-squares sense. The relatively poor agreement for $\mathrm{m}=0$ and $\mathrm{St}=0.2$ is attributable to non-parallel effects. 
On the whole, Figure 4 indicates very good agreement between theory and experiment up to about 4-5 diameters downstream of the nozzle exit. Further confirmation of the theory was given by comparing the radial decay and phase velocity of the signals [31]. Ryu et al. [47] have recently provided additional comparisons between linear stability theory and LESdetermined wave packets.

The good agreement between linear theory and experiment for wave packets in the initial shear layer development does not necessarily indicate that linear theory is sufficient for directly predicting the acoustic field associated with the wave packets. This is because the far field, at a given frequency, depends on the entire wave packet, which, at least at lower frequencies, extends well beyond the end of the potential core. It appears that, at a minimum, non-parallel and non-linear wave interactions need to be accounted for to predict the wave packet evolution further downstream of the linear region. This can be done via large scale simulations such as DNS or even LES but the associated cost can become prohibitive for realistic flow conditions and so a lower order approach is needed. The Parabolized Stability Equations (PSE) represents a compromise. Originally developed by Herbert [48], the PSE method expands the domain of linear stability methods by relaxing the parallel-flow assumption as well as allowing for the inclusion of non-linear terms. The method has been used with considerable success in the prediction of convectively unstable flows such as boundary layers [49], jet flows [50] and planar mixing layers with chemistry [51]. PSE computations are usually started using some known mean flow, typically a laminar boundary layer solution. In the present modeling, we instead use the time-averaged turbulent flow, which can be predicted to suitable accuracy with existing RANS techniques provided by TTC.

The development here follows the traditional PSE analysis (see Bertolotti \& Herbert [49]) where we decompose the flow field

$$
q(x, r, \theta, t)=\left[u_{x}, u_{r}, u_{\theta}, T, \rho\right]^{T}
$$

into its mean and fluctuating parts,

$$
q=Q+q^{\prime}
$$

where the prime denotes the fluctuations about the mean state,

$$
Q(x, r)=\left[\bar{u}_{x}, \bar{u}_{r}, 0, \bar{T}, \bar{\rho}\right]^{T}
$$

We further decompose the fluctuations as

$$
q^{\prime}=\sum_{m, n} \hat{q}_{m n}(x, r) \epsilon_{m n} e^{i\left(\int^{x} \alpha(\xi) d \xi+m \theta-n \omega t\right)}
$$

where the real and imaginary parts of alpha denote axial wave number and growth rate, respectively; $m$ is the azimuthal wave number and omega is the temporal frequency while epsilon represents initial amplitude and phase of mode $(m, n)$.

Substituting this decomposition into the Navier-Stokes equations we obtain

$$
\begin{gathered}
\mathcal{L}_{m n} \hat{q}_{m n}+\mathcal{M} \frac{\partial \hat{q}_{m n}}{\partial x}+\mathcal{N} \frac{\partial \hat{q}_{m n}}{\partial r}=\ldots \\
\frac{1}{R e} \mathcal{V}_{m n} \hat{q}_{m n}+\frac{\mathcal{F}_{m n}\left(q^{\prime}\right)}{\epsilon_{m n}} \exp \left(-i \int^{x} \alpha(\xi) d \xi\right)
\end{gathered}
$$

where the operators

$$
(\mathcal{L}, \mathcal{M}, \mathcal{N}, \mathcal{V})_{m n}
$$

are linear in $\mathrm{q}$, while the operator $\mathrm{F}$ represents non-linear terms. We discretize the resulting equations using fourth-order finite differences in the radial direction, closing the equations with characteristic boundary conditions. Initial conditions come from linear stability analysis where we use the mean flow at the nozzle. To this end we solve the compressible Rayleigh equation,

$$
\begin{gathered}
\frac{1}{r} \frac{d}{d r}\left(r \frac{d p_{m}}{d r}\right)-\left(\frac{2 \bar{u}_{r}}{\alpha \bar{u}-\omega}+\frac{\bar{\rho}^{\prime}}{\bar{\rho}}\right) \frac{d p_{m}}{d r}-\ldots \\
\left(\frac{m^{2}}{r^{2}}+\alpha^{2}-\bar{\rho}(\alpha \bar{u}-\omega)^{2}\right) p_{m}=0 .
\end{gathered}
$$

where $p_{m}$ is the pressure eigenmode and other variables are as defined above. To march the solution downstream we discretize the stream-wise derivative using a 1st-order implicit Euler difference. This code is implemented in Matlab. ${ }^{\mathrm{TM}}$

The solution of the linear PSE has been implemented in cylindrical coordinates. Using the parallel-flow linear solution at the nozzle exit as initial, the solution is iterated in the downstream direction. The mean flow field used in analysis is measured experimentally using PIV as reported in [31]. The LPSE provides relative phase and amplitude evolution, but lacks the absolute phase and amplitude at the nozzle exit needed to match the pressure predictions along the hydrodynamic array. To determine these amplitudes we use a simpler form of the 

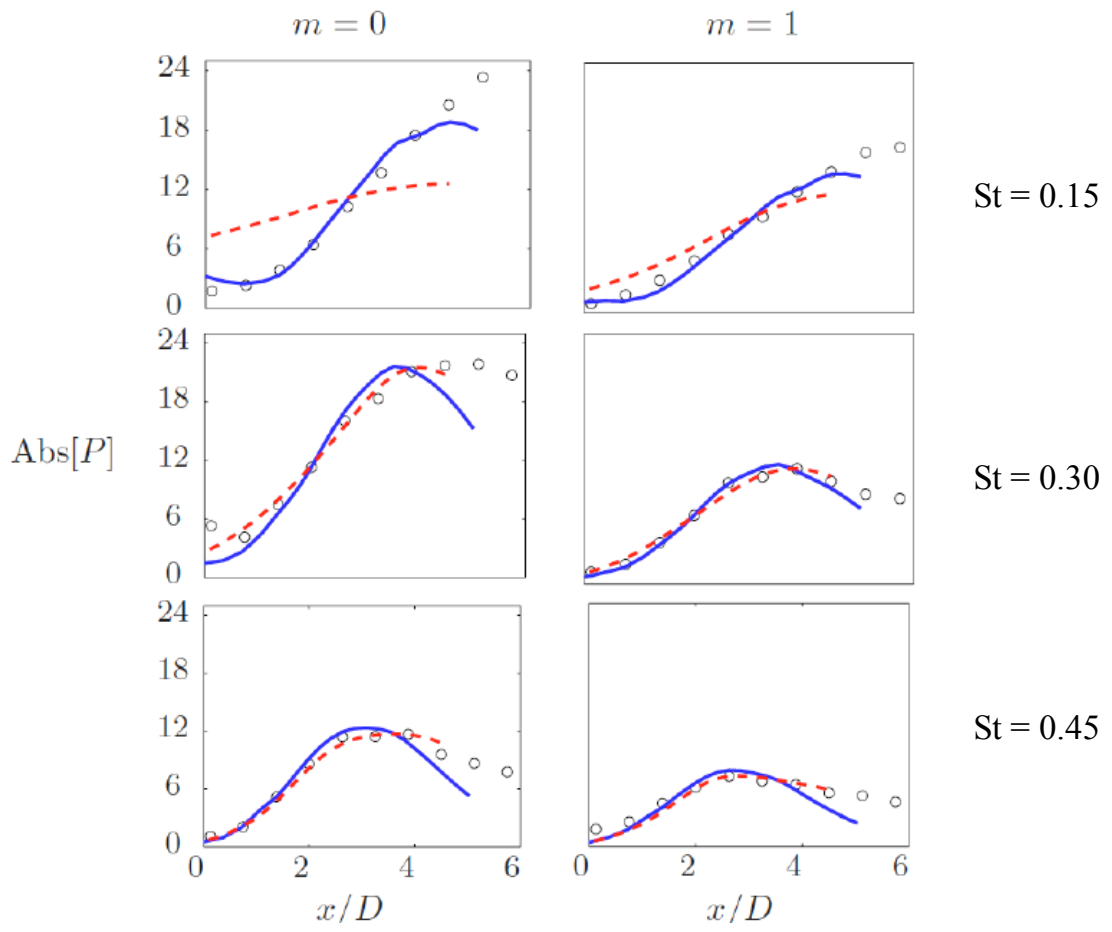

Figure 5. Comparison of microphone near-field array measurements from [31] (symbols) with predictions of linear stability analysis [31] (red line) and present linear PSE (blue line) of a cold Mach $=0.9$ iet.

instability-wave beamforming procedure introduced by Suzuki \& Colonius [52] by minimizing the squared error between the microphone data and the LPSE, using all eight microphones. Figure 5 shows the wavepacket pressure amplitude obtained with a Mach 0.9 cold jet (corresponding to set-point 7 in the study by Tanna [53]), for azimuthal wave numbers $\mathrm{m}=0$ and 1 , and Strouhal numbers $0.15,0.30$ and 0.45 . This figure includes the results of linear stability analysis (LST). Note the significant improvement offered by LPSE over LST at St $=$ 0.15 , particularly for $\mathrm{m}=0$, illustrating clearly the importance of non-parallel effects. For higher frequencies the differences between LST and LPSE mostly disappear; at these frequencies the disturbance wavelengths are such that non-parallel effects, the only distinction between LPSE and LST, are of vanishing importance.

In order to evaluate the predictive capability for a supersonic jet, we consider a Mach 1.5 perfectly expanded cold jet (setpoint B118 in [53]) which was recently measured in the UTRC ART with the rotating microphone array discussed in the previous section. The mean flow field needed for the calculations was provided by TTC Technologies using a RANS method with a k- $\varepsilon$ turbulence model. The results, shown in
Figure 6, indicate that the predictions are similarly good as for the subsonic jets. This appears to partly contradict the results of Ryu \& Lele [47] who found their supersonic jet near-field predictions were not as successful compared to their subsonic predictions. However, the results in the current case are mixed; in some instances the predictions are excellent (e.g. $\mathrm{St}=0.3, \mathrm{~m}$ $=1$ ), while others are not as close (e.g. St $=0.3, \mathrm{~m}=0$ ), with the trend being towards overshoot followed by rapid decay. This is somewhat surprising, given the slower spread rate of the supersonic jet shear layer, placing less stress on the PSE Ansatz. However, the slow spread of the jet allows the exponential growth of the modes to continue further than it would otherwise. This follows from the linear simulation approach where the only mechanism available for arresting growth is shear layer spread. We hypothesize that the inclusion of non-linear terms will address this problem.

We note that the linear results here are independent of the initial amplitudes of instability waves, and we have simply adjusted the amplitude to give best agreement, for a given frequency, with the microphone measurements. For a predictive model, the spectra of incident disturbances will need to be specified, as discussed below in Section 3.4 

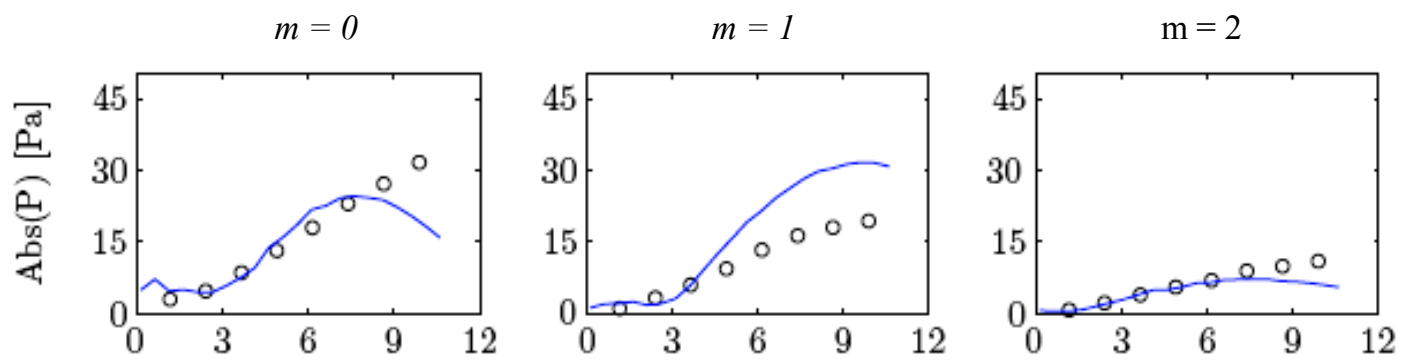

$\mathrm{St}=0.1$
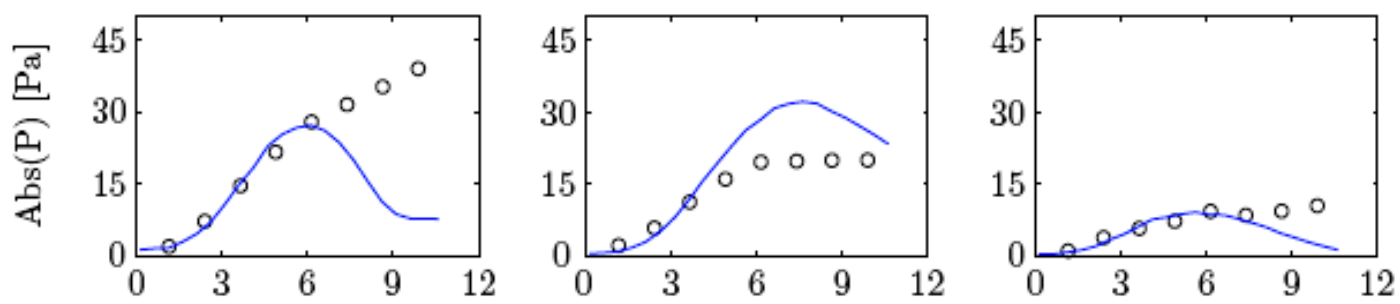

$\mathrm{St}=0.2$
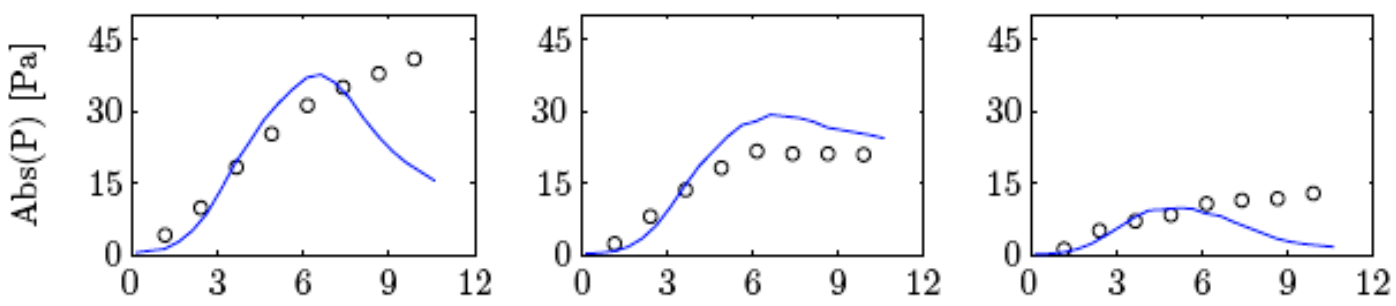

$\mathrm{St}=0.3$
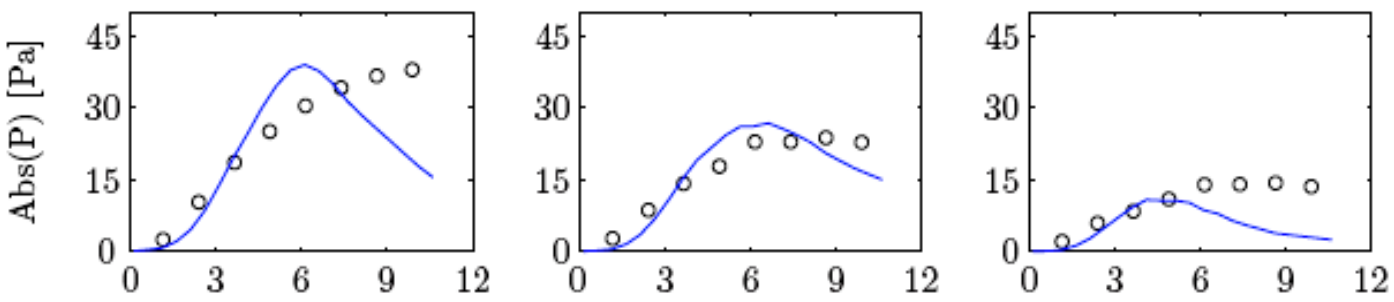

$\mathrm{St}=0.4$
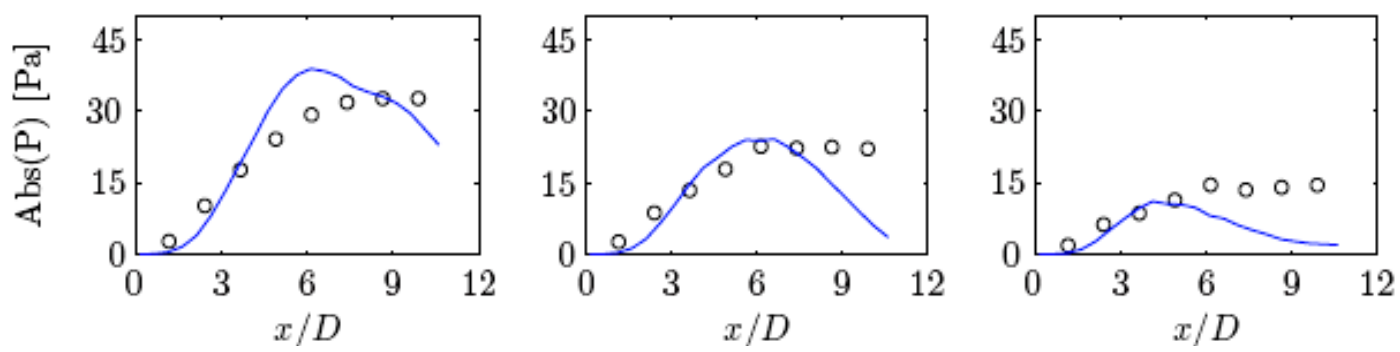

$\mathrm{St}=0.5$

Figure 6. Phased-array microphone measurements (symbols) compared with predictions of linear PSE (blue line) analysis of a cold Mach $=1.5$ jet.

\subsection{Nonlinear Wave packet models}

The Nonlinear PSE (NPSE) method allows for energy transfer between frequency modes and, in particular, it allows for the development of a zero-frequency mode that can alter mean flow development. As such, the resulting mean flow includes the effects of the fluctuating organized waves modeled by PSE but it does not include the effects of small-scale turbulence.
The PSE method breaks down when these smaller-scale fluctuations become significant. From a computational perspective, the jet flow can be approximately decomposed into three sections; one without interactions of wave packets (their evolution being well approximated via LST or linear PSE), another one with interactions but weak turbulence (NPSE sufficient), and a third region where interactions are still important but turbulent fluctuations are significant (no low 


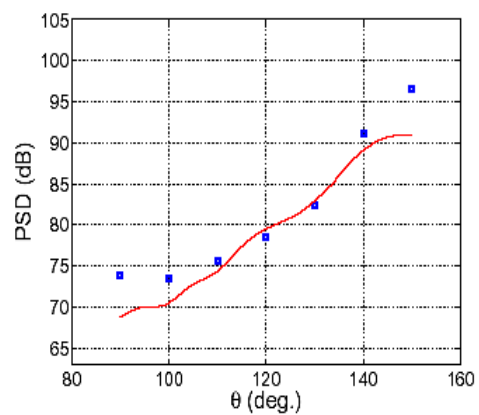

(a) $\mathrm{B} 118 ; \mathrm{St}=0.4$

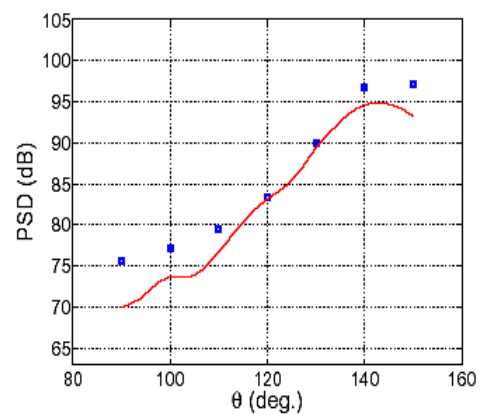

(b) $\mathrm{B} 120 ; \mathrm{St}=0.4$

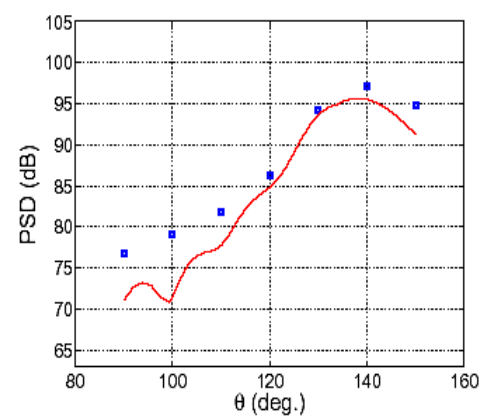

(c) $\mathrm{B} 122 ; \mathrm{St}=0.4$

Figure 7. Comparison of measured (symbols) and projected (solid) sound pressure at $\mathrm{St}=0.4$ along far field array for B118, B120, and B122 at St=0.4

order method is available). Work is currently underway to extend the validity of the NPSE method into the third region by the incorporation of turbulence models.

\subsection{Far-field sound predicted from wave packets.}

Ultimately the wave packet models developed above will be used to predict far-field sound. Recall that the location of the microphone arrays is chosen to be just outside the shear layer where pressure fluctuations are decaying and linearized equations hold. The far-field pressure at the associated frequencies may be predicted by the straight forward projection method described in [29]. Essentially, the jet is enclosed in a Kirchhoff surface, and the far-field pressure is related, via integration with an appropriate Green's function, to the pressure data along the surface.

In order to validate the projection method for the supersonic jet cases considered here, simultaneous near-field and far-field pressure measurements were acquired for an ideally expanded jet operating at $\mathrm{M}_{\mathrm{j}}=1.5$ over a range of temperature ratios, as summarized in Table 1. For the proof-of concept study reported here, the array was designed to address frequencies in the immediate neighborhood of $\mathrm{St}=0.4$, generally considered to be the peak frequency for broadband jet noise and Mach wave emission from the initial mixing region (i.e. $x=D_{j}<10$ ). This Strouhal number corresponds to the spectral peaks in Figure 2a.

Table 1. Set Points Considered

\begin{tabular}{ccc}
\hline Set pt. & $M_{\infty}\left(M_{\text {jet }}\right)$ & $T_{\text {jet }} / T_{\infty}$ \\
\hline$B 118$ & $1.50(1.5)$ & $1.00($ cold $)$ \\
\hline$B 120$ & $1.77(1.5)$ & $1.40($ hot $)$ \\
\hline$B 122$ & $1.98(1.5)$ & $1.74($ hot $)$ \\
\hline
\end{tabular}

Wave-numbers associated with significantly higher frequencies are under-resolved by the array microphone spacing reported here, while for significantly lower frequencies, the array does not extend far enough down-stream for meaningful projection results. Variants of the current design with different objectives will be considered in on-going applications.

The far-field data does not allow decomposition into individual azimuthal modes, and thus our comparison is based on the total acoustic field. Figure 7 compares projection results against far-field sideline array measurements as a function of inlet directivity angle, theta. The projection includes azimuthal modes $0, \pm 1$, and \pm 2 . Overall agreement between measurement and projection is very good. Discrepancies apparent for $\theta>$ 140 and for $\theta<115$ most likely result from insufficient extent of the array, and are most pronounced at lower (sideline) angles, where fine-scale turbulence noise is believed to be dominant. The latter observation suggests that, for $\mathrm{St}=0.4$, a significant portion of fine-scale mixing noise is generated down-stream of $x / D_{j}=10$.

It is interesting to note that, despite the significant increase in jet velocity, the peak pressure amplitude at this frequency is relatively constant, while the pressure amplitude at moderate angles (e.g. $130 \mathrm{deg}$.) increases by roughly $15 \mathrm{~dB}$. This trend is accurately captured by the projection method, and is perhaps most naturally explained as a shift in the Mach angle toward smaller (more sideward) angles due to the increased convection speed. A similar 'broadening' of the directivity pattern has been observed in subsonic jets with heating. In that case, convection speeds were approximately constant, and the broadening was attributed to contraction of the wave-packet scale. Thus, it is possible that changes in the correlation scale also play a role in explaining the far-field trends. Detailed parameter studies aimed at elucidating the role of these parameters for both subsonic and supersonic jets are in progress and will be reported in a forthcoming paper. 


\subsection{Outlook and future studies}

While work continues on refining wave packet models and farfield projection procedures, as well as gathering additional experimental data for validation, the results presented seem sufficiently promising to conclude that the noise radiated by large-scale structures in turbulent jets may be modeled using PSE and projected to the far-field using a Kirchhoff surface approach. A key enabler in this procedure is the development of near-field microphone arrays capable of providing the pressure statistics needed to validate models. Our framework provides, for the first time, a deterministic model that will allow understanding and predicting noise radiated by largescale turbulence.

One area for refinement is the use of nonlinear PSE to better capture the downstream evolution of the wave packets. In nonlinear calculations, the amplitude of the overall wave packet is not arbitrary, and the spectra of incident disturbances at the nozzle lip must be specified. Current studies underway are evaluating the sensitivity of the nonlinear results to the specified spectrum. Once fully validated, these nonlinear PSE methods can then be used for parametric studies associated with introducing disturbances at the nozzle lip in order to reduce far-field sound, thus providing strategies for active noise control.

\subsection{ACKNOWLEDGEMENTS}

The authors gratefully acknowledge the support of TTC Technologies Inc., the SBIR lead partner in this NAVAIR sponsored work.

\subsection{REFERENCES}

[1] Hileman, J. and Samimy, M., "Effects of Vortex Generating Tabs on Noise Sources in an Ideally Expanded Mach 1.3 Jet," $8^{\text {th }}$ AIAA/CEAS Aeroacoustics Conference, Breckenridge, Colorado, June 2002, AIAA 2002-2482.

[2] Gupta, A., Haber, L., Graziosi, P., Kirtley, K., Martens, S. and Majjigi, M., "Jet Noise Reduction Technology Development for the F414/F404," Proceedings of the $17^{\text {th }}$ Annual ONR Propulsion Meeting, Monterey, California, June 2004.

[3] Seiner, J.M., Ukeiley, L.S. and Bernard, J.J., "AeroPerformance Efficient Noise Reduction for the F404-400 Engine," $11^{\text {th }}$ AIAA/CEAS Aeracoustics Conference, Monterey, California, May 2005, AIAA 2005-3048.
[4] Alkislar, B.M. and Butler, G.W., "Significant Improvements on Jet Noise Reduction by ChevronMicrojet Combination," $13^{\text {th }}$ AIAA/CEAS Aeroacoustics Conference, Rome, Italy, May 2007, AIAA 2007-3598.

[5] Marten, S. and Haber, L., 2008, "Jet Noise Reduction for High Speed Exhaust Systems," Proceedings of the ASME Turbo Expo 2008, Berlin, Germany, June 2008, GT2008-50455.

[6] Schlinker, R.H., Simonich, J.C., Reba, R.A., Colonius, T., Gudmundsson, K., Ladeinde, F. and Cai, X., "Supersonic Jet Noise from Round and Chevron Nozzles; Experimental and Numerical Studies," $15^{\text {th }}$ AIAA/CEAS Aeroacoustic Conference, Miami, Florida, June 2009, AIAA 2009-3257.

[7] Greska, B., Krothapalli, A., Seiner, J.M., Jansen, B. and Ukeiley, L., "The Effects of Microjet Injection on an F404 Jet engine," $11^{\text {th }}$ AIAA/CEAS Aeroacoustics Conference, May 2005, AIAA 2005-3047.

[8] Haber, L., Gupta, A., Kirtley, K., Martens, S. and Hoelle, S., "Jet Noise Reduction Technology Development for the F414/F404," Proceedings of the 18th Annual ONR Propulsion Meeting, Monterrey, California, August, 2005.

[9] Callender, B., Gutmark, E. and Martens, S., "Comprehensive Study of the Fluidic Injection Technology for Jet Noise Reduction," 13th AIAA/CEAS Aeroacoustics Conference, Rome, Italy, May 2007, AIAA 2007-3608.

[10] Wlezein, R.W. and Kibens, V., "Influence of Nozzle Asymmetry on Supersonic Jets," AIAA Journal, Vol. 26, No. 1, January 1988.

[11] Viswanathan, K., "Adaption of the Beveled Nozzle for the F/A-18 Noise Reduction," Proceedings of the $18^{\text {th }}$ Annual ONR Propulsion Meeting, Monterey, California, August 2005.

[12] Cowan, S.J. and Crouch, R.W., "Transmission of Sound Through a Two-Dimensional Shielding Jet," Progress in Aeronautics and Astronautics, Vol. 37, Publisher: AIAA, pp. 173-190, 1975.

[13] Goodykoontz, J., "Effect of a Semi-annular Thermal Acoustic Shield on Jet Exhaust Noise," NASA TM 81615, 1980. 
[14] Majjigi, R.K., Brausch, J.F., Janardan, B.A., Balsa, T.F., Knott, P.R. and Pickup, N., "Free Jet Feasibility Study of a Thermal Acoustic Shield Concept for AST/VCE Application," NASA CR 3758, 1984.

[15] Schlinker, R.H., Liljenberg, S.A., Polak, D.R., Post, K.A., Chipman, C.T. and Stern, A.M., "Supersonic Jet Noise Source Characteristics and Propagation: Engine and Model Scale," $13^{\text {th }}$ AIAA/CEAS Aeroacoustics Conference, Rome, Italy, May 2007, AIAA 2007-3623.

[16] Davis, C.L., Calkins, F.T. and Butler, G.W., "High Frequency Jet Nozzle Actuators for Noise Reduction," SPIE Proceedings, Vol. 5054, pp. 34-44, 2003.

[17] Butler, G.W. and Calkins, F.T., "Initial Attempts to Suppress Jet Noise Using Piezoelectric Actuators," $9^{\text {th }}$ AIAA/CEAS Aeroacoustics Conference, Hilton Head, South Carolina, May 2003, AIAA 2-003-3192.

[18] Samimy, M., Kim, J.H., Kasnter, J., Adamovich, I. and Utkin, Y., "Active Control of a Mach 0.9 Jet for Noise Mitigation Using Plasma Actuators," AIAA Journal, Vol. 45, No. 4, April 2007.

[19] Kim, J.H., Adamovich, I. and Samimy, M., "Active Noise Control in a Mach 1.3 Ideally-Expanded Jet with Plasma Actuators," 46 ${ }^{\text {th }}$ AIAA Aerospace Sciences Meeting, Reno, Nevada, January 2008, AIAA 2008-38.

[20] Bodony, D.J. and Lele, S.K., "Current Status of Jet Noise Predictions Using Large-Eddy Simulation," AIAA Journal, Vol. 46, No. 2, February 2008.

[21] Goldstein, M.E., "A Generalized Acoustic Analogy," J. Fluid Mech., Vol. 488, pp. 315-333, 2003.

[22] Goldstein, M.E. and Leib, S.J., "The Role of Instability Waves in Predicting Jet Noise," J. Fluid Mech., Vol. 525, pp. 37-72, 2005.

[23] Goldstein, M.E. and Leib, S.J., "The Aeroacoustics of Slowly Diverging Supersonic Jets," J. Fluid Mech., Vol. 600, pp. 291-337, 2008.

[24] Karabasov, S., Afsar, M., Hynes, T. and Dowling, A., "Using Large Eddy Simulation with an Acoustic Analogy Approach for Jet Noise Modeling," $14^{\text {th }}$ AIAA/CEAS Aeroacoustics Conference, Vancouver, BC, Canada, May 2008, AIAA 2008-2985.

[25] Tam, C.K.W., "Supersonic Jet Noise," Annual Rev. Fluid Mech., Vol. 27, pp. 17-43, 1995.
[26] Hussain, A.K.M.F., "Coherent Structures and Turbulence,” J. Fluid Mech., Vol. 173, pp. 303-356, 1986.

[27] Tam, C. K. W., Golebiowski, M. and Seiner, J. M., "On the Two Components of Turbulent Mixing Noise from Supersonic Jets," $2^{\text {nd }}$ AIAA/CAES Aeroacoustics Conference, State College, PA, May 1996, AIAA 961716.

[28] Tam, C.K.W., "Jet Noise: Since 1952," Theoretical and Computational Fluid Dynamics, Vol. 10, pp. 393-405, 1998

[29] Reba, R., Simonich, J.C. and Schlinker, R.H., "Measurement of Source Wave-Packets in High-Speed Jets and Connection to Far Field," $14^{\text {th }}$ AIAAA/CEAS Aeroacoustics Conference, Vancouver, Canada, May 2008, AIAA 2008-2891.

[30] Reba, R.A., Narayanan, S., Colonius, T. and Suzuki, T., "Modeling Jet Noise from Organized Structures Using Near Field Hydrodynamic Pressure", $11^{\text {th }}$ AIAA/CEAS Aeroacoustics Conference, Monterey, CA, May 2005, AIAA 2005-3093.

[31] Suzuki, T. and Colonius, T., "Instability Waves in a Subsonic Round Jet Detected using a Near-Field Phased Microphone Array," J. Fluid Mech., Vol. 565, pp. 197226, 2006.

[32] Simonich, J.C., Narayanan, S. and Schlinker, R.H., "Facility and Data Quality Issues for Scale-Model Jet Noise Testing," $41^{\text {st }}$ Aerospace Sciences Meeting, Reno, NV, January 2003, AIAA2003-1057

[33] Schlinker, R.H., Simonich, J.C. and Reba, R.A., "Decomposition of High Speed Jet Noise: Source Characteristics and Propagation Effects," AIAA/CEAS Aeroacoustics Conference, Vancouver, Canada, May 2008, AIAA 2008-2890.

[34] Tam, C.K.W. and Burton, D.E., "Sound Generated by Instability Waves of Supersonic Flows," Part 2, Axisymmetric Jets," J. Fluid Mech., Vol. 138, pp. 273295, 1984.

[35] Tam, C.K.W., "Supersonic Jet Noise," Annual Rev. Fluid Mech., Vol. 27, pp.17-43, 1995.

[36] Blumen, W., "Shear Layer Instability of an Inviscid Compressible Fluid," J. Fluid Mech., Vol., 40, pp. 769$781,1970$. 
[37] Brown, G. L. and Roshko, A., "On Density Effects and Large Structure in Turbulent Mixing Layers," J. Fluid Mech., Vol. 64, pp. 775-816, 1974.

[38] Liu, J. T. C., "Developing Large-scale Wavelike Eddies and the Near Jet Noise Field," J. Fluid Mech., Vol. 62, pp. 437-464, 1974.

[39] Michalke, A. and Fuchs, H. V., "On Turbulence and Noise of an Axisymmetric Shear Flow," J. Fluid Mech., Vol. 70, pp. 179-205, 1975.

[40] Fuchs, H. V. and Michel, U., "Experimental Evidence of Turbulent Source Coherence Affecting Jet Noise," AIAA J., Vol. 16, pp. 871-872, 1978.

[41] Maestrello, L. and Fung, Y.T., "Quasi-Periodic Structure of a Turbulent Jet," J. Sound Vib., Vol. 64, pp. 107-122, 1979.

[42] Mankbadi, R. R. and Liu, J. T. C., "A study of the Interactions Between Large-Scale Coherent Structures and Fine-Grained Turbulence in a Round Jet," Phil. R. Soc. London, Vol. 298, pp. 541-602, 1981.

[43] Mankbadi, R. R., "On the Interaction Between Fundamental and Subharmonic Instability Waves in a Turbulent Round Jet," J. Fluid Mech., Vol. 160, pp. 385419, 1985.

[44] Crighton, D. G. and Huerre, P., "Shear-Layer Pressure Fluctuations and Superdirective Acoustic Sources," J. Fluid Mech., Vol. 220, pp. 355-368, 1990.

[45] Bridges, J. and Brown, C., "Parametric Testing of Chevrons on Single Flow Hot Jets," $10^{\text {th }}$ AIAA/CEAS Aeroacoustics Conference, Manchester, UK, May 2004, AIAA 2004-2824.

[46] Bridges, J. and Wernet, M., "Measurements of the Aeroacoustic Sound Source in Hot Jets," $9^{\text {th }}$ AIAA/CEAS Aeracoustics Conference, Hilton Head, SC, May 2003, AIAA 2003-3130.
[47] Ryu, J., Lele, S.K. and Viswanathan, K., "Investigation of the Role of Instability Waves in Jet Noise Radiation," $46^{\text {th }}$ AIAA Aerospace Sciences Meeting, Reno, NV, January 2008, AIAA 2008-26.

[48] Herbert, T., "Parabolized Stability Equations," Annul. Rev. Fluid Mech., Vol. 29, pp. 245-283, 1997.

[49] Bertolotti, F. P. and Herbert, T., "Analysis of the Linear Stability of Compressible Boundary Layers Using the PSE," Theoretical and Computational Fluid Dynamics, Vol. 3, pp. 117-124, 1991.

[50] Malik, M. R. and Chang, C. L., "PSE applied to Supersonic Jet Instability," $35^{\text {th }}$ AIAA Aerospace Sciences Meeting, Reno, NV, January 1997, AIAA 19970758 .

[51] Day, M. J., Mansour, N. N. and Reynolds, W. C., "Nonlinear Stability and Structure of Compressible Reacting Mixing Layers, J. Fluid Mech. Vol. 446, pp. 375-408, 2001.

[52] Suzuki, T. and Colonius, T., "Identification of Jet Instability Waves and Design of a Microphone Array," $10^{\text {th }}$ AIAA/CEAS Aeroacoustics Conference, Manchester, United Kingdom, May 2004, AIAA 2004-2960.

[53] Tanna, H.K., Dean P. D. and Burrin, R. H., "The Generation and Radiation of Supersonic Jet Noise, Part III, Turbulent Mixing Noise Data," AFAPL-TR-76-65, 1976.

[54] Gudmundsson, K. and Colonius T., "Spatial Stability Analysis of Chevron Jet Profiles," $13^{\text {th }}$ AIAA/CEAS Aeroacoustics Conference, Rome, Italy, May 2007, AIAA 2007-3599. 
